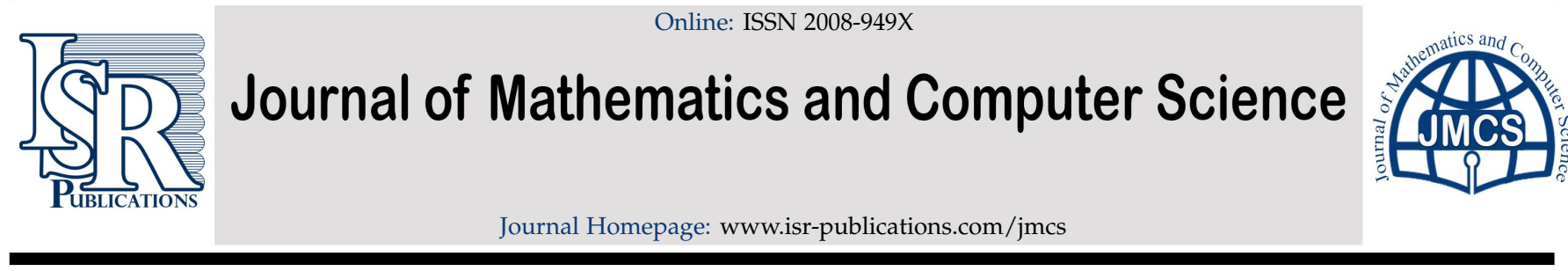

\title{
Fractional calculus formulas for Mathieu-type series and generalized Mittag-Leffler function
}

\author{
Owais Khan ${ }^{\mathrm{a}}$, Serkan Araci $^{\mathrm{b}, *}$, Mohd Saif ${ }^{\mathrm{a}}$ \\ ${ }^{a}$ Department of Applied Mathematics, Aligarh Muslim University, Aligarh-202002, India. \\ ${ }^{b}$ Department of Economics, Faculty of Economics, Administrative and Social Sciences, Hasan Kalyoncu University, TR-27410 \\ Gaziantep, Turkey.
}

\begin{abstract}
Fractional calculus is allowing integrals and derivatives of any positive order (the term 'fractional' kept only for historical reasons), which can be considered a branch of mathematical physics which mainly deals with integro-differential equations, where integrals are of convolution form with weakly singular kernels of power-law type. In recent decades fractional calculus has won more and more interest in applications in several fields of applied sciences. In this line, our main object to investigate image formulas of generalized fractional hypergeometric operators involving the product of Mathieu-type series and generalized Mittag-Leffler function. We also consider some interesting special cases of derived results by specializing suitable value of the parameters.
\end{abstract}

Keywords: Fractional calculus operators, Mathieu-type series, generalized Mittag-Leffler function, Fox-Wright function.

2010 MSC: 26A33, 33C20, 33B15.

(C)2020 All rights reserved.

\section{Introduction}

Fractional calculus is a generalization of ordinary differentiation and integration to arbitrary noninteger order. The subject is as old as the differential calculus, and goes back to the time when Leibnitz and Newton invented differential calculus. The idea of fractional calculus has been a subject of interest not only among mathematicians, but also among physicists and engineers. During the past few years, a super deal of interest in existence of solution to several classes of fractional differential equations has been shown. In particular, various researchers investigated the existences of mild solution of fractional differential equations with nonlocal conditions. For instance, fractional differential equations with nonlocal conditions are often used for modeling various phenomena arising in control, electrochemistry, viscoelastic, and electromagnetic. We refer the reader to the papers $[11,27,42]$ and cited therein. Since last few decades, many authors like Saigo [30], Saigo and Maeda [31], Srivastava and Saxena [37], Kilbas

\footnotetext{
*Corresponding author

Email addresses: owkhan05@gmail.com (Owais Khan), mtsrkn@hotmail.com (Serkan Araci), usmanisaif153@gmail.com (Mohd Saif)
}

doi: $10.22436 /$ jmcs.020.02.05

Received: 2019-07-18 Revised: 2019-09-05 Accepted: 2019-09-17 
and Saigo, etc., have extensively studied the properties, applications, and extensions of various fractional integral and differential operators of FC. It is notable that Gurmeet et al. [34] established the images of Mathieu type series of thereby providing an extension of several earlier results due to Kilbas and Sebastian [18]. Thus, many authors have explored new approach of applications by making use of FC operators to investigate image formula involving special functions of one and more variables, which are useful in the problem of applied science in recent years, such as fractional diffusion, fractional reaction, fractional stochastic theory, dynamical systems theory and anomalous diffusion in complex systems, etc., for example, see, $[1,2,4,5,12-16,19,23-25,28,29,37]$.

Throughout this paper we have used $\mathbb{C}, \mathbb{R}, \mathbb{R}^{+}, \mathbb{Z}_{0}^{+}$and $\mathbb{N}$ to be the set of complex number, real and positive real numbers, positive set of integers and set of natural numbers, respectively.

We recall the generalized hypergeometric fractional integrals and derivatives, introduced by Marichev [19] and later extended by Saigo and Maeda [31]. These operators are known as the Marichev-SaigoMaeda operators. The generalized FC operators involving the Appell function or the Horn $F_{3}(\cdot)$ function in the kernel are defined as follows:

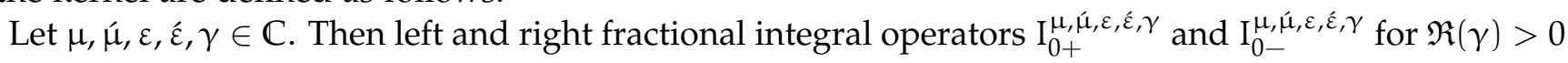
are respectively defined by (see [31]):

$$
\left(I_{0+}^{\mu, \mu ́ \varepsilon, \hat{\varepsilon}, \gamma_{f}}\right)(u)=\frac{u^{-\mu}}{\Gamma(\gamma)} \int_{0}^{u}(u-t)^{\gamma-1} t^{-\mu} F_{3}(\mu, \hat{\mu}, \varepsilon, \hat{\varepsilon} ; \gamma ; 1-t / u, 1-u / t) f(t) d t
$$

and

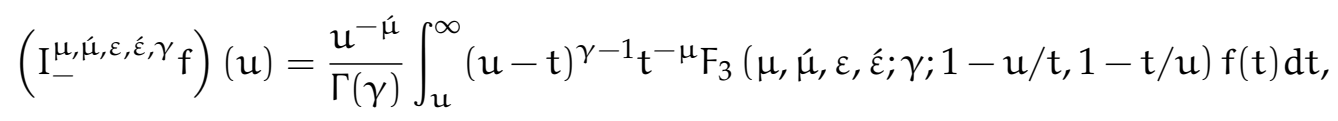

where $F_{3}$ is one of the Appell series defined by (see [36])

$$
F_{3}(\mu, \mu, \varepsilon, \hat{\varepsilon} ; \gamma ; u, v)=\sum_{m, n=0}^{\infty} \frac{(\mu)_{m}(\hat{\mu})_{\mathfrak{n}}(\varepsilon)_{m}(\varepsilon)_{\mathfrak{n}}}{(\gamma)_{m+n}} \frac{u^{m}}{m !} \frac{v^{n}}{n !}, \quad(\max \{|u|,|v|\}<1) .
$$

These operators reduce to the following Saigo fractional integral operators (see [30]):

$$
\left(\mathrm{I}_{0+}^{\mu+\varepsilon, 0,-\tau, 0, \mu} \mathrm{f}\right)(\mathrm{u})=\left(\mathrm{I}_{0+}^{\mu, \varepsilon, \tau} \mathrm{f}\right)(\mathrm{u}), \quad(\gamma \in \mathbb{C}),
$$

and

$$
\left(\mathrm{I}_{-}^{\mu+\varepsilon, 0,-\tau, 0, \mu_{f}}\right)(\mathrm{u})=\left(\mathrm{I}_{-}^{\mu, \varepsilon, \tau} f\right)(\mathrm{u}), \quad(\gamma \in \mathbb{C}) .
$$

Let $\mu, \hat{\mu}, \varepsilon, \varepsilon, \gamma \in \mathbb{C}$ with $\mathfrak{R}(\gamma)>0$ and $u \in \mathbb{R}^{+}$. Then left and right generalized fractional differential operators $\mathrm{D}_{0+}^{\mu, \hat{\mu}, \varepsilon, \hat{\varepsilon}, \gamma}$ and $\mathrm{D}_{0-}^{\mu, \hat{\mu}, \varepsilon, \hat{\varepsilon}, \gamma}$, respectively, for $\mathfrak{R}(\gamma)>0$ involving the Appell function $\mathrm{F}_{3}$ in the kernel are defined as follows (see [31]):

$$
\begin{aligned}
& \left(D_{0+}^{\mu, \mu, \varepsilon, \hat{\varepsilon}, \gamma_{f}}\right)(u)=\left(I_{0+}^{-\hat{\mu},-\mu,-\hat{\varepsilon},-\varepsilon, \gamma} f\right)(u) \\
& =\left(\frac{\mathrm{d}}{\mathrm{du}}\right)^{\mathfrak{n}}\left(\mathrm{I}_{0+}^{-\mu},-\mu,-\hat{\varepsilon}+\mathrm{n},-\varepsilon, \gamma+\mathrm{n}_{\mathrm{f}}\right)(\mathrm{u}) \quad(\mathfrak{R}(\gamma)>0 ; \mathfrak{n}=[\mathfrak{R}(\gamma]+1) \\
& =\frac{1}{\Gamma(n-\gamma)}\left(\frac{d}{d u}\right)^{n}\left(u^{-\mu}\right) \int_{0}^{u}(u-t)^{n-\gamma-1} t^{-\mu} \\
& \times F_{3}(-\mu,-\mu, n-\varepsilon,-\varepsilon, n-\gamma ; 1-t / u, 1-u / t) f(t) d t
\end{aligned}
$$

and

$$
\begin{aligned}
& \left(D_{-}^{\mu, \hat{\mu}, \varepsilon, \hat{\varepsilon}, \gamma_{f}}\right)(\mathrm{u})=\left(\mathrm{I}_{-}^{-\mathrm{m}^{\prime} u,-\mu,-\hat{\varepsilon},-\varepsilon, \gamma_{f}}\right)(\mathrm{u})
\end{aligned}
$$

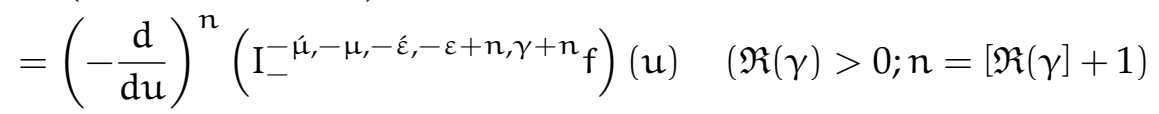




$$
\begin{aligned}
= & \frac{1}{\Gamma(n-\gamma)}\left(-\frac{d}{d u}\right)^{n}\left(u^{-\hat{\lambda}}\right) \int_{u}^{\infty}(u-t)^{n-\gamma-1} t^{-\mu} \\
& \times F_{3}(-\mu,-\mu, n-\varepsilon,-\varepsilon, n-\gamma ; 1-u / t, 1-t / u) f(t) d t .
\end{aligned}
$$

These operators reduce to the following Saigo fractional differential operators (see [30]):

$$
\left(D_{0+}^{\mu+\varepsilon, 0,-\tau, 0, \mu} f\right)(u)=\left(D_{0+}^{\mu, \varepsilon, \tau} f\right)(u), \quad(\gamma \in \mathbb{C})
$$

and

$$
\left(D_{-}^{\mu+\varepsilon, 0,-\tau, 0, \mu} f\right)(u)=\left(D_{-}^{\mu, \varepsilon, \tau} f\right)(u), \quad(\gamma \in \mathbb{C}) .
$$

Furthermore, we also have the following useful relation to prove our main results (see [31, p.394]):

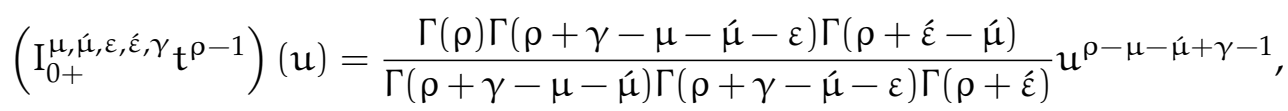

$$
\begin{aligned}
& (\mu, \hat{\mu}, \varepsilon, \hat{\varepsilon}, \gamma \in \mathbb{C} \text { with } \mathfrak{R}(\gamma)>0 \text { and } \mathfrak{R}(\rho)>\max \{0, \mathfrak{R}(\mu+\hat{\mu}-\gamma), \mathfrak{R}(\dot{\mu}-\dot{\varepsilon})\})
\end{aligned}
$$

and

$$
\begin{aligned}
& \left(\mathrm{I}_{0,-}^{\mu, \dot{\mu}, \varepsilon, \hat{\varepsilon}, \gamma_{\mathrm{t}} \rho-1}\right)(\mathrm{u})=\frac{\Gamma(1+\mu+\hat{\mu}-\gamma-\rho) \Gamma(1+\mu+\hat{\varepsilon}-\gamma-\rho) \Gamma(1-\varepsilon-\rho)}{\Gamma(1-\rho) \Gamma(1+\mu+\hat{\mu}+\hat{\varepsilon}-\gamma-\rho) \Gamma(1+\mu-\varepsilon-\rho)} \mathrm{u}^{\rho-\mu-\tilde{\mu}+\gamma-1}, \\
& (\mu, \hat{\mu}, \varepsilon, \hat{\varepsilon}, \gamma \in \mathbb{C} ; \mathfrak{R}(\gamma)>0 \text { and } \mathfrak{R}(\rho)<1+\min \{\mathfrak{R}(-\varepsilon), \mathfrak{R}(\mu+\mu-\gamma), \mathfrak{R}(\mu+\hat{\varepsilon}-\gamma)\}) .
\end{aligned}
$$

The generalized Wright hypergeometric function ${ }_{r} \Psi_{s}[x]$ also called Fox-Wright function $([8,44])$ is defined as:

$$
\begin{aligned}
{ }_{r} \Psi_{s}[x] & ={ }_{r} \Psi_{s}\left[\begin{array}{c}
\left(\gamma_{1}, \hat{\gamma}_{1}\right), \ldots,\left(\gamma_{r}, \hat{\gamma}_{s}\right) ; \\
\left(l_{1}, \hat{l}_{1}\right), \ldots,\left(l_{r}, \hat{l}_{s}\right) ;
\end{array}\right]=\sum_{k=0}^{\infty} \frac{\Gamma\left(\gamma_{1}+\hat{\gamma}_{1} k\right), \ldots, \Gamma\left(\gamma_{r}+\hat{\gamma}_{s} k\right)}{\Gamma\left(l_{1}+\hat{l}_{1} k\right), \ldots, \Gamma\left(l_{r}+\hat{l}_{s} k\right)} \frac{x^{k}}{k !} \\
& =H_{r, s+1}^{1, r}\left[-x \mid\left(1-\gamma_{1}, \gamma_{1}\right), \ldots,\left(1-\gamma_{r}, \gamma_{r}\right)(0,1),\left(1-l_{1}, \ell_{1}^{\prime}\right), \ldots,\left(1-l_{s}, l_{s}^{\prime}\right)\right],
\end{aligned}
$$

where $H_{r, s+1}^{1, r}[x]$ denotes the Fox-H function [8], coefficients $\gamma_{1}^{\prime}, \ldots, \gamma_{r}^{\prime}, l_{1}^{\prime}, \ldots, l_{s}^{\prime} \in \mathbb{R}^{+}$, and the series absolutely converges for all $x \in \mathbb{C}$ when $1+\sum_{j=1}^{s} l_{j}^{\prime}-\sum_{m=1}^{r} \gamma_{m}^{\prime}>0$.

The Mathieu series appeared in the study of elasticity of solid bodies in the work of Emile Leonard Mathieu. Since then numerous authors have studied various type of problems arising from the Mathieu series in several diverse ways.

The following familiar infinite series was introduced by Mathieu [20] as

$$
S(l)=\sum_{k=1}^{\infty} \frac{2 k}{\left(k^{2}+l^{2}\right)} \quad\left(l \in \mathbb{R}^{+}\right) .
$$

Integral representation of (1.11) is given by (see [7])

$$
S(l)=\frac{1}{l} \int_{0}^{\infty} \frac{t \sin (l t)}{e^{t}-1} d t
$$

Numerous interesting problems and solutions handling with integral representations and bound for the following generalization of the Mathieu series with fractional power

$$
S_{\sigma}(l)=\sum_{k=1}^{\infty} \frac{2 k}{\left(k^{2}+l^{2}\right)^{\sigma}} \quad\left(l \in \mathbb{R}^{+} ; \sigma \leqslant 1\right)
$$

can be found in the works by Cerone and Lenard [6] and Tomovski and Trencevski [41]. Succeeding the work of Cerone and Lenard [6], Srivastava and Tomovski in [38] defined a family of generalized Mathieu 
series

$$
S_{\sigma}^{(\alpha, \beta)}(l, d)=S_{\sigma}^{(\alpha, \beta)}\left(l,\left\{d_{k}\right\}_{k=1}^{\infty}\right)=\sum_{k=1}^{\infty} \frac{2 d_{k}^{\beta}}{\left(d_{k}^{\alpha}+l^{2}\right)^{\sigma}} \quad\left(l, d, \alpha, \beta, \sigma \in \mathbb{R}^{+}\right),
$$

where the positive sequence $d=\left\{d_{k}\right\}_{k=1}^{\infty}=\left\{d_{1}, d_{2}, \ldots\right\} \quad\left(\lim _{k \rightarrow \infty} d_{k}=\infty\right)$ is to prefer the infinite series $\sum_{k=1}^{\infty} \frac{1}{d_{k}^{\sigma \alpha-\beta}}$ is convergent.

In the sequel Tomovski and Mehrez [39] proposed a generalization of definition (1.12) in the following power series

$$
S_{\sigma, \tau}^{(\alpha, \beta)}(l, d ; u)=S_{\sigma, \tau}^{(\alpha, \beta)}\left(l,\left\{d_{k}\right\}_{k=1}^{\infty} ; u\right)=\sum_{k=1}^{\infty} \frac{2 d_{k}^{\beta}(\tau)_{k}}{\left(d_{k}^{\alpha}+l^{2}\right)^{\sigma}} \frac{u^{k}}{k !} \quad\left(l, d, \alpha, \beta, \sigma \in \mathbb{R}^{+} ;|u| \leqslant 1\right) .
$$

Evidently, the case $d_{k}=k, \alpha=2, \beta, \tau=1$ and $\sigma$ with $\sigma+1$ corresponds to the Mathieu series defined by Tomovski and Pogány [40] of the form

$$
S_{\sigma+1,1}^{(2,1)}(l, k ; u)=S_{\sigma}(l ; u)=\sum_{k=1}^{\infty} \frac{2 k u^{k}}{\left(k^{2}+l^{2}\right)^{\sigma+1}} \quad\left(l, \sigma \in \mathbb{R}^{+} ;|u| \leqslant 1\right) .
$$

In recent decades, the Mittag-Leffler function plays an important role in several branches of mathematics and engineering sciences, such as statistics, chemistry, mechanics, quantum physics, informatics and others. In particular, it is an explicit formula for the resolvent of Riemann-Liouville fractional integrals by Hille and Tamarkin. On this and similar formulas many results are based still for solving fractional integral and differential equations. For numerous applications of the Mittag-Leffler function to fractional calculus see [1-4, 13-16, 24, 26, 29]. Due to many useful applications it was crowned by Goreno and Mainardi in [9] as a Queen function of Fractional Calculus. Besides fractional calculus the Mittag-Leffler function also plays an important role in various branches of applied mathematics and engineering sciences, such as chemistry, biology, statistics, thermodynamics, mechanics, quantum physics, informatics, signal processing and others.

For our present investigation, we also need to recall the following definitions: Gosta Mittag-Leffler in 1903 introduced the so-called Mittag-Leffler function (see [22])

$$
\mathrm{E}_{\delta}(z)=\sum_{\mathrm{r}=0}^{\infty} \frac{z^{\mathrm{r}}}{\Gamma(\delta \mathrm{r}+1)} \quad(\mathfrak{R}(\delta)>0 ; z \in \mathbb{C}) .
$$

We choose to recall some of extensions of the Mittag-Leffler function. Wiman [43] introduced

$$
\mathrm{E}_{\delta, v}(z)=\sum_{\mathrm{r}=0}^{\infty} \frac{z^{\mathrm{r}}}{\Gamma(\delta \mathrm{r}+\boldsymbol{v})} \quad(\min \{\mathfrak{R}(\delta), \mathfrak{R}(\nu)\}>0 ; z \in \mathbb{C})
$$

Prabhakar [26] presented

$$
E_{\delta, v}^{\eta}(z)=\sum_{r=0}^{\infty} \frac{(\eta)_{r} z^{r}}{\Gamma(\delta r+v) r !} \quad(\min \{\mathfrak{R}(\delta), \mathfrak{R}(v)\}>0, \eta ; z \in \mathbb{C}),
$$

where $(\lambda)_{v}$ denotes the Pochhammer symbol which is defined (for $\lambda, v \in \mathbb{C}$ ), in terms of Gamma function as:

$$
(\lambda)_{v}:=\frac{\Gamma(\lambda+v)}{\Gamma(\lambda)}= \begin{cases}1, & (v=0 ; \lambda \in \mathbb{C} \backslash\{0\}), \\ \lambda(\lambda+1) \cdots(\lambda+n-1), & (v=n \in \mathbb{N} ; \lambda \in \mathbb{C}),\end{cases}
$$

it is understood conventionally that $(0)_{0}:=1$. 
Shukla and Prajapati [33] gave further extension of Mittag-Laffler function as:

$$
E_{\delta, v}^{\eta, q}(z)=\sum_{r=0}^{\infty} \frac{(\eta)_{q r} z^{r}}{\Gamma(\delta r+v) r !} \quad(\min \{\mathfrak{R}(\eta), \mathfrak{R}(\delta), \mathfrak{R}(v)\}>0 ; q \in(0,1) \cup \mathbb{N} ; z \in \mathbb{C}) .
$$

Due to the great importance of fractional calculus operators involving various types of special functions, in this paper, we establish certain integral and derivation formulas of the product of Mathieu-type series and generalized Mittag-Leffler function by using generalized fractional hypergeomrtric operators. Moreover, we also find out some special cases of our main results.

\section{Fractional integral formulas}

In this section, we establish fractional integral formulas involving the product of generalized MittagLeffler function and Mathieu-type series using left and right Marichecv-Saigo-Maeda operators, which are expressed in terms of Fox-Wright function under the given conditions of (1.10).

Theorem 2.1. Let $\mu, \hat{\mu}, \varepsilon, \hat{\varepsilon}, \gamma, \rho \in \mathbb{C}$ be such that $\mathfrak{R}(\sigma+\lambda k+\zeta r)>\max \{0 ; \mathfrak{R}(\mu+\hat{\mu}+\varepsilon-\lambda k \gamma)\} \mathfrak{R}(\mu-\lambda k-$ $\zeta r-\varepsilon)>0, \mathfrak{R}(\rho)>0, \mathfrak{R}(\delta)>0$ and $\alpha, \beta, \sigma, l \in \mathbb{R}^{+}, \mathrm{q} \in(0,1) \cup \mathbb{N}$. Then left fractional integral formula holds true:

$$
\begin{aligned}
& \left\{I_{0+}^{\mu, \hat{\mu}, \varepsilon, \hat{\varepsilon}, \gamma}\left(t^{\rho-1} S_{\sigma, \tau}^{(\alpha, \beta)}\left(l, d ; t^{\lambda}\right) E_{\delta, v}^{\eta, q}\left(z t^{\zeta}\right)\right\}(u)\right. \\
& =\frac{u^{\rho+\gamma-\mu-\hat{\mu}-1}}{\Gamma(\eta)} S_{\sigma, \tau}^{(\alpha, \beta)}\left(l, d ; u^{\lambda}\right) \\
& \times{ }_{4} \Psi_{4}\left[\begin{array}{c}
(\rho+\gamma-\mu-\mu-\varepsilon+\lambda k, \zeta),(\rho+\varepsilon-\mu+\lambda k, \zeta),(\rho+\lambda k, \zeta),(\eta, q) \\
(\rho+\gamma-\mu-\mu+\lambda k, \zeta),(\rho+\varepsilon+\lambda k, \zeta),(\rho+\gamma+\varepsilon-\mu+\lambda k, \zeta),(\nu, \delta)
\end{array} \mid z u^{\zeta}\right] .
\end{aligned}
$$

Proof. To prove the above result, using (1.13) and (1.14) as series form, and then arranging the order of integration and summation (which is valid under the given condition of Theorem 2.1), left hand side of (2.1) becomes

$$
\begin{aligned}
\left\{I_{0+}^{\mu, \tilde{\mu}, \varepsilon, \hat{\varepsilon}, \gamma}\left(t^{\rho-1} S_{\sigma, \tau}^{(\alpha, \beta)}\left(l, d ; t^{\lambda}\right) E_{\delta, \gamma}^{\eta, q}\left(z t^{\zeta}\right)\right\}(u)=\right. & \sum_{k=1}^{\infty} \frac{2 d_{k}^{\beta}(\tau)_{k}}{\left(d_{k}^{\alpha}+l^{2}\right)^{\sigma}} \frac{1}{k !} \\
& \times \sum_{r=0}^{\infty} \frac{(\eta)_{q r} z^{r}}{\Gamma(\delta r+v) r !}\left\{I_{0,+}^{\mu, \tilde{\mu}, \varepsilon, \varepsilon, \gamma}\left(t^{\rho+\lambda k+\zeta r-1}\right)\right\}(u) .
\end{aligned}
$$

Applying (1.8), we have

$$
\begin{aligned}
& =\sum_{k=1}^{\infty} \frac{2 d_{k}^{\beta}(\tau)_{k}}{\left(d_{k}^{\alpha}+l^{2}\right)^{\sigma}} \frac{1}{k !} \sum_{r=0}^{\infty} \frac{(\eta)_{q r} z^{r}}{\Gamma(\delta r+v) r !} \times \frac{\Gamma(\rho+\lambda k+\zeta r) \Gamma(\rho+\lambda k+\zeta r+\gamma-\mu-\mu-\varepsilon)}{\Gamma(\rho+\lambda k+\zeta r+\varepsilon) \Gamma(\rho+\gamma-\mu-\mu+\lambda k+\zeta r)} \\
& \times \frac{1}{\Gamma(\tau)} \frac{\Gamma(\rho+\hat{\varepsilon}-\mu+\lambda \hat{k}+\zeta r)}{\Gamma\left(\rho+\lambda k+\zeta r+\gamma-\mu^{\prime}-\varepsilon\right)} \frac{z^{r}}{r !} u^{\rho+\gamma-\mu-\mu ́}+\lambda k+\zeta r-1 .
\end{aligned}
$$

Finally, solving the above expression, in view of using definition (1.10), we achieve the required result (2.1).

In view of the relation (1.1), we construct the consequence of Theorem 2.1.

Corollary 2.2. Let $\mu, \varepsilon, \gamma, \rho \in \mathbb{C}$ be such that $\mathfrak{R}(\sigma+\lambda k+\zeta \mathrm{r})>\max \{0 ; \mathfrak{R}(\varepsilon-\gamma)\} \mathfrak{R}(\rho)>0, \mathfrak{R}(\delta)>0$ and $\alpha, \beta, \sigma, l \in \mathbb{R}^{+}, q \in(0,1) \cup \mathbb{N}$. Then following holds true:

$$
\begin{aligned}
& \left\{I_{0+}^{\mu, \epsilon, \gamma}\left(t^{\rho-1} S_{\sigma, \tau}^{(\alpha, \beta)}\left(l, d ; t^{\lambda}\right) E_{\delta, \nu}^{\eta, q}\left(z t^{\zeta}\right)\right\}(u)\right. \\
& =\frac{u^{\rho-\varepsilon-1}}{\Gamma(\eta)} S_{\sigma, \tau}^{(\alpha, \beta)}\left(l, d ; u^{\lambda}\right) \times{ }_{3} \Psi_{3}\left[\begin{array}{c}
(\rho+\gamma-\varepsilon+\lambda k, \zeta),(\rho+\lambda k, \zeta),(\eta, q) \\
(\rho+\lambda k-\varepsilon, \zeta),(\rho+\lambda k+\gamma+\mu, \zeta),(v, \delta)
\end{array} \mid z u^{\zeta}\right] .
\end{aligned}
$$


Theorem 2.3. Let $\mu, \tilde{\mu}, \varepsilon, \hat{\varepsilon}, \gamma, \rho \in \mathbb{C}$ be such that $\mathfrak{R}(\sigma+\lambda k-\zeta r)<1+\min \{0 ; \mathfrak{R}(\mu+\hat{\mu}-\eta), \mathfrak{R}(\mu+\hat{\varepsilon}-\eta)$, $\mathfrak{R}(-\varepsilon)\}, \mathfrak{R}(\rho)>0, \mathfrak{R}(\delta)>0$ and $\alpha, \beta, \sigma, l \in \mathbb{R}^{+}, \mathrm{q} \in(0,1) \cup \mathbb{N}$. Then the following right fractional integral formula holds true:

$$
\begin{aligned}
& \left\{I_{0-}^{\mu, \mu ́ \varepsilon, \varepsilon, \gamma}\left(t^{\rho-1} S_{\sigma, \tau}^{(\alpha, \beta)}\left(l, d ; t^{\lambda}\right) E_{\delta, \gamma}^{\eta, q}\left(z t^{-\zeta}\right)\right\}(u)\right. \\
& =\frac{u^{\rho+\gamma-\mu-\tilde{\mu}-1}}{\Gamma(\eta)} S_{\sigma, \tau}^{(\alpha, \beta)}\left(l, d ; u^{\lambda}\right) \\
& \times{ }_{4} \Psi_{4}\left[\begin{array}{c}
(1+\mu+\mu-\gamma-\rho-\lambda k, \zeta),(1+\mu+\varepsilon-\gamma-\rho-\lambda k, \zeta),(1-\epsilon-\rho-\lambda k, \zeta),(\eta, q) \\
(1+\mu+\mu+\varepsilon-\gamma-\lambda k, \zeta)(1+\mu-\epsilon-\rho-\lambda k, \zeta),(1-\rho-\lambda k, \zeta),(\gamma, \delta)
\end{array} \mid z u^{\zeta}\right] .
\end{aligned}
$$

Proof. To prove that above result, using (1.13) and (1.14) as series form, and than arranging the order of integration and summation (which is valid under the given condition of Theorem 2.3), left hand side of (2.2) becomes

$$
\begin{aligned}
\left\{I_{0-}^{\mu, \hat{\mu}, \varepsilon, \hat{\varepsilon}, \gamma}\left(t^{\rho-1} S_{\sigma, \tau}^{(\alpha, \beta)}\left(l, d ; t^{\lambda}\right) E_{\delta, v}^{\eta, q}\left(z t^{-\zeta}\right)\right\}(u)=\right. & \sum_{k=1}^{\infty} \frac{2 d_{k}^{\beta}(\tau)_{k}}{\left(d_{k}^{\alpha}+l^{2}\right)^{\sigma}} \frac{1}{k !} \\
& \times \sum_{r=0}^{\infty} \frac{(\eta)_{q r} z^{r}}{\Gamma(\delta r+v) r !}\left\{I_{0,-}^{\mu, \hat{\mu}, \varepsilon, \hat{\varepsilon}, \gamma}\left(t^{\rho+\lambda k+\zeta r-1}\right)\right\}(u) .
\end{aligned}
$$

Now, applying the relation (1.2), we have

$$
\begin{aligned}
= & \sum_{k=1}^{\infty} \frac{2 d_{k}^{\beta}(\tau)_{k}}{\left(d_{k}^{\alpha}+l^{2}\right)^{\sigma}} \frac{1}{k !} \sum_{r=0}^{\infty} \frac{(\eta)_{q r} z^{r}}{\Gamma(\delta r+v) r !} \\
& \times \frac{\Gamma\left(1+\mu+\mu^{\prime}-\gamma-\rho-\lambda k+\zeta r\right) \Gamma(1+\mu+\mu+\varepsilon-\gamma-\rho-\lambda k+\zeta r)}{\Gamma(1+\mu+\mu+\varepsilon-\gamma-\lambda k+\zeta r) \Gamma(1+\mu-\varepsilon-\lambda k+\zeta r)} \\
& \times \frac{1}{\Gamma(\tau)} \frac{\Gamma(1-\epsilon-\rho-\lambda k+\zeta r)}{\Gamma\left(1+\mu-\varepsilon^{\prime}-\rho-\lambda k+\zeta r\right)} \frac{z^{r}}{r !} u^{\rho+\gamma+\mu-\mu+\lambda k-\zeta r-1} .
\end{aligned}
$$

Finally, solving the above expression with the help of (1.10), we achieve the required result (2.2).

In view of the relation (1.2), we get the following consequence of Theorem 2.3.

Corollary 2.4. Let $\mu, \varepsilon, \gamma, \rho \in \mathbb{C}$ be such that $\mathfrak{R}(\sigma+\lambda k+\zeta r)<1+\min \{0 ; \mathfrak{R}(\varepsilon), \mathfrak{R}(\gamma)\}, \mathfrak{R}(\rho)>0, \mathfrak{R}(\delta)>0$ and $\alpha, \beta, \sigma, l \in \mathbb{R}^{+}, q \in(0,1) \cup \mathbb{N}$. Then following formula holds true:

$$
\begin{aligned}
& \left\{I_{0-}^{\mu, \varepsilon, \gamma}\left(t^{\rho-1} S_{\sigma, \tau}^{(\alpha, \beta)}\left(l, d ; t^{\lambda}\right) E_{\delta, v}^{\eta, q}\left(z t^{-\zeta}\right)\right\}(u)\right. \\
& =\frac{u^{\rho-\varepsilon-1}}{\Gamma(\eta)} S_{\sigma, \tau}^{(\alpha, \beta)}\left(l, d ; u^{\lambda}\right) \times{ }_{3} \Psi_{3}\left[\begin{array}{c}
(1-\rho-\lambda k+\varepsilon, \zeta),(1-\rho-\lambda k+\gamma, \zeta),(\eta, q) \\
(1-\rho-\lambda k, \zeta)(1-\rho-\lambda k+\gamma+\mu+\varepsilon, \zeta),(\nu, \delta)
\end{array} \mid z u^{\zeta}\right] .
\end{aligned}
$$

\section{Fractional derivative formulas}

Here, we compute fractional derivative formulas involving product of generalized Mittag-Leffler function and Mathieu-type series using left and right Marichecv-Saigo-Maeda operators, which are expressed in terms of Fox-Wright function under the given conditions of (1.10).

Theorem 3.1. Let $\mu, \hat{\mu}, \varepsilon, \varepsilon, \gamma, \rho \in \mathbb{C}$ be such that $\mathfrak{R}(\sigma+\lambda k+\zeta r)>\max \{0 ; \mathfrak{R}(\gamma-\mu+\hat{\mu}+\varepsilon), \mathfrak{R}(\varepsilon-\mu)\}$, $\mathfrak{R}(\rho), \mathfrak{R}(\delta)>0$ and $\alpha, \beta, \sigma, l \in \mathbb{R}^{+}, \mathrm{q} \in(0,1) \cup \mathbb{N}$. Then following formula holds true:

$$
\begin{aligned}
\{ & D_{0+}^{\mu, \hat{\mu}, \varepsilon, \hat{\varepsilon}, \gamma}\left(\mathrm{t}^{\rho-1} S_{\sigma, \tau}^{(\alpha, \beta)}\left(l, d ; t^{\lambda}\right) E_{\delta, \nu}^{\eta, q}\left(z t^{\zeta}\right)\right\}(u) \\
= & \frac{u^{\rho+\gamma-\mu-\mu}-1}{\Gamma(\eta)} S_{\sigma, \tau}^{(\alpha, \beta)}\left(l, d ; u^{\lambda}\right) \\
& \times{ }_{4} \Psi_{4}\left[\begin{array}{c}
(\rho-\gamma+\mu+\mu+\varepsilon+\lambda k, \zeta),(\rho-\hat{\varepsilon}+\mu+\lambda k, \zeta),(\rho+\lambda k, \zeta),(\eta, q) \\
(\rho-\gamma+\mu-\mu+\lambda k, \zeta)(\rho-\varepsilon+\lambda k, \zeta),(\rho-\gamma+\mu-\mu+\lambda k, \zeta),(\nu, \delta)
\end{array} \mid z u^{\zeta}\right] .
\end{aligned}
$$


Proof. In order to prove that above result, using (1.3) and (1.14) as series form, and then arranging the order of integration and summation (which is valid under the given condition of Theorem 3.1), left hand side of (3.1) becomes

$$
\begin{aligned}
\left\{D_{0+}^{\mu, \dot{\mu}, \varepsilon, \dot{\varepsilon}, \gamma}\left(\mathrm{t}^{\rho-1} S_{\sigma, \tau}^{(\alpha, \beta)}\left(l, d ; t^{\lambda}\right) E_{\delta, \nu}^{\eta, q}\left(z t^{\zeta}\right)\right\}(u)=\right. & \sum_{k=1}^{\infty} \frac{2 d_{k}^{\beta}(\tau)_{k}}{\left(d_{k}^{\alpha}+l^{2}\right)^{\sigma}} \frac{1}{k !} \\
& \times \sum_{r=0}^{\infty} \frac{(\eta)_{q r} z^{r}}{\Gamma(\delta r+v) r !}\left\{I_{0,+}^{\mu, \mu, \varepsilon, \varepsilon, \gamma}\left(t^{\rho+\lambda k+\zeta r-1}\right)\right\}(u) .
\end{aligned}
$$

Applying the result (1.6), we get

$$
\begin{aligned}
= & \sum_{k=1}^{\infty} \frac{2 d_{k}^{\beta}(\tau)_{k}}{\left(d_{k}^{\alpha}+l^{2}\right)^{\sigma}} \frac{1}{k !} \sum_{r=0}^{\infty} \frac{(\eta)_{q r} z^{r}}{\Gamma(\delta r+v) r !} \times \frac{1}{\Gamma(\tau)} \frac{\Gamma(\rho-\varepsilon+\mu+\lambda k+\zeta r)}{\Gamma\left(\rho+\lambda k+\zeta r-\gamma+\mu^{\prime}-\varepsilon^{\prime}\right)} \frac{z^{r}}{r !} u^{\rho-\gamma+\mu+\mu^{\prime}+\lambda k+\zeta r-1} \\
& \times \frac{\Gamma(\rho+\lambda k+\zeta r) \Gamma(\rho+\lambda k+\zeta r-\gamma+\mu+\mu-\varepsilon)}{\Gamma(\rho+\lambda k+\zeta r-\varepsilon) \Gamma(\rho-\gamma+\mu+\mu+\lambda k+\zeta r)} .
\end{aligned}
$$

Finally, using Definition (1.10), we achieve the desired result (3.1).

According to the relation (1.6), we get the following consequence of Theorem 3.1.

Corollary 3.2. Let $\mu, \varepsilon, \gamma, \rho \in \mathbb{C}$ be such that $\mathfrak{R}(\sigma+\lambda k+\zeta r)>\min \{0 ; \mathfrak{R}(\mu+\varepsilon+\gamma)\}, \mathfrak{R}(\rho)>0, \mathfrak{R}(\delta)>0$ and $\alpha, \beta, \sigma, l \in \mathbb{R}^{+}, q \in(0,1) \cup \mathbb{N}$. Then following relation holds true:

$$
\begin{aligned}
& \left\{\begin{array}{l}
D_{0+\varepsilon}^{\mu, \varepsilon, \gamma}\left(t^{\rho-1} S_{\sigma, \tau}^{(\alpha, \beta)}\left(l, d ; t^{\lambda}\right) E_{\delta, v}^{\eta, q}\left(z t^{\zeta}\right)\right\}(u) \\
\quad=\frac{u^{\rho+\varepsilon-1}}{\Gamma(\eta)} S_{\sigma, \tau}^{(\alpha, \beta)}\left(l, d ; u^{\lambda}\right) \times{ }_{3} \Psi_{3}\left[\begin{array}{c}
(\rho+\lambda k+\gamma+\mu+\varepsilon, \zeta),(\rho+\lambda k+\gamma, \zeta),(\eta, q) \\
(\rho+\lambda k+\gamma, \zeta)(\rho+\lambda k+\varepsilon, \zeta),(v, \delta)
\end{array} \mid z u^{\zeta}\right] .
\end{array}\right.
\end{aligned}
$$

Theorem 3.3. Let $\mu, \tilde{\mu}, \varepsilon, \varepsilon, \gamma, \rho \in \mathbb{C}$ be such that $\mathfrak{R}(\sigma+\lambda k+\zeta r)<1+\min \{0 ; \mathfrak{R}(\gamma-\mu+\mu), \mathfrak{R}(\gamma-\mu-\varepsilon)$, $\mathfrak{R}(\varepsilon)\} \mathfrak{R}(\rho)>0$ and $\mathfrak{R}(\delta)>0, \alpha, \beta, \sigma, l \in \mathbb{R}^{+}, \mathrm{q} \in(0,1) \cup \mathbb{N}$. Then following holds true:

$$
\begin{aligned}
& \left\{D_{0-}^{\mu, \mu, \varepsilon, \hat{\varepsilon}, \gamma}\left(t^{\rho-1} S_{\sigma, \tau}^{(\alpha, \beta)}\left(l, d ; t^{\lambda}\right) E_{\delta, \nu}^{\eta, q}\left(z t^{-\zeta}\right)\right\}(u)\right. \\
& =\frac{u^{\rho+\mu+\mu}-\gamma-1}{\Gamma(\eta)} S_{\sigma, \tau}^{(\alpha, \beta)}\left(l, d ; u^{\lambda}\right) \\
& \quad \times{ }_{4} \Psi_{4}\left[\begin{array}{c}
(1-\rho+\gamma-\mu-\mu-\lambda k, \zeta),(1-\rho+\gamma-\mu-\lambda k, \zeta),(1-\rho+\varepsilon-\lambda k, \zeta),(\eta, q) \\
(1-\rho+\gamma-\mu-\mu-\epsilon-\lambda k, \zeta)(1-\rho-\lambda k, \zeta),(1-\rho-\mu+\varepsilon+\varepsilon-\lambda k, \zeta),(\nu, \delta)
\end{array} \mid z u^{\zeta}\right] .
\end{aligned}
$$

Proof. Applying (1.14) and (1.5), and then arranging the order of integration and summation (which is valid under the condition of Theorem 3.3), left hand side of (3.2) can be writen as

$$
\begin{aligned}
\left\{D_{0-}^{\mu, \tilde{\mu}, \varepsilon, \dot{\varepsilon}, \gamma}\left(\mathrm{t}^{\rho-1} S_{\sigma, \tau}^{(\alpha, \beta)}\left(l, d ; t^{\lambda}\right) E_{\delta, v}^{\eta, q}\left(z t^{-\zeta}\right)\right\}(u)=\right. & \sum_{k=1}^{\infty} \frac{2 d_{k}^{\beta}(\tau)_{k}}{\left(d_{k}^{\alpha}+l^{2}\right)^{\sigma}} \frac{1}{k !} \\
& \times \sum_{r=0}^{\infty} \frac{(\eta)_{q r} z^{r}}{\Gamma(\delta r+v) r !}\left\{I_{0,+}^{\mu, \tilde{\mu}, \varepsilon, \hat{\varepsilon}, \gamma}\left(t^{\rho+\lambda k+\zeta r-1}\right)\right\}(u) .
\end{aligned}
$$

Now in view of (1.4) and (1.9), we obtain the following expression

$$
\begin{aligned}
& =\sum_{k=1}^{\infty} \frac{2 d_{k}^{\beta}(\tau)_{k}}{\left(d_{k}^{\alpha}+l^{2}\right)^{\sigma}} \frac{1}{k !} \sum_{r=0}^{\infty} \frac{(\eta)_{q r} z^{r}}{\Gamma(\delta r+v) r !} \times \frac{\Gamma(1-\rho-\mu-\mu-\lambda k+\zeta r) \Gamma(1-\rho-\mu-\varepsilon-\lambda k+\gamma+\zeta r)}{\Gamma(1-\rho-\mu-\mu-\varepsilon-\lambda k+\zeta r) \Gamma(1-\rho-\mu+\varepsilon+\lambda k+\zeta r)} \\
& \times \frac{1}{\Gamma(\tau)} \frac{\Gamma(1-\rho+\varepsilon-\lambda k+\zeta r)}{\Gamma(1-\rho-\lambda k-\zeta r)} \frac{z^{r}}{r !} u^{\rho+\mu+\mu-\gamma-1} .
\end{aligned}
$$

Solving the above expression with the help of (1.10), we achieve the desired result (3.2). 
In view of the relation (1.7), we get the following consequence of Theorem 3.3.

Corollary 3.4. Let $\mu, \varepsilon, \gamma, \rho \in \mathbb{C}$ be such that $\mathfrak{R}(\sigma+\lambda k+\zeta r)<1+\min \{0 ; \mathfrak{R}(-\varepsilon-\mathfrak{R}(\mu)-1, \mathfrak{R}(\gamma+\mu)\}$, $\mathfrak{R}(\rho)>0, \mathfrak{R}(\delta)>0$ and $\alpha, \beta, \sigma, l \in \mathbb{R}^{+}, \mathrm{q} \in(0,1) \cup \mathbb{N}$. Then following relation holds:

$$
\begin{aligned}
& \left\{D_{0-}^{\mu, \varepsilon, \gamma}\left(\mathrm{t}^{\rho-1} S_{\sigma, \tau}^{(\alpha, \beta)}\left(\mathrm{l}, \mathrm{d} ; \mathrm{t}^{\lambda}\right) \mathrm{E}_{\delta, \nu}^{\eta, \mathrm{q}}\left(z \mathrm{t}^{-\zeta}\right)\right\}(\mathrm{u})\right. \\
& \quad=\frac{\mathrm{u}^{\rho+\varepsilon-1}}{\Gamma(\eta)} S_{\sigma, \tau}^{(\alpha, \beta)}\left(\mathrm{l}, \mathrm{d} ; \mathrm{u}^{\lambda}\right) \times{ }_{3} \Psi_{3}\left[\begin{array}{c}
(1-\rho-\lambda k+\gamma+\mu, \zeta),(1-\rho-\lambda k-\varepsilon \zeta),(\eta, q) \\
(1-\rho-\lambda k+\gamma-\mu-\varepsilon, \zeta)(1-\rho-\lambda k, \zeta),(\nu, \delta)
\end{array} \mid z u^{\zeta}\right] .
\end{aligned}
$$

\section{Conclusion}

In this research, we investigate four image formulas of generalized fractional hypergeometric (of Marichev-Saigo-Maeda) operators involving the product of Mathieu-type series and generalized MittagLeffler function in the kernel, which are expressed in terms of Fox-Wright function. The results presented in this paper are extensions of the known results given by various authors (see, e.g., $[2,21,32,34]$ ). Moreover, the results derived in this paper correspond to Saigo hypergeometric fractional calculus operators as special cases and it can be easily seen that, if we set $\varepsilon=-\mu$ and $\varepsilon=0$ in (1.1) and (1.2), they yield the Erdelyi-Kober, the Riemann-Liouville, and the Weyl fractional integral and derivative operators. Thereby, the results presented here can also be obtained corresponding to the above well known fractional operators. Therefore, the results derived in this article would at once give way a large number of results involving a many diversity of special functions occurring in the problems of mathematical physics, science, and engineering, etc..

\section{References}

[1] P. Agarwal, J. J. Nieto, Some fractional integral formulas for the Mittag-Leffler type function with four parameters, Open math., 13 (2015), 537-546. 1, 1

[2] S. Ahmed, On the generalized fractional integrals of the generalized Mittag-Leffler function, SpringerPlus, 3 (2014), 5 pages. 1,4

[3] S. Araci, G. Rahman, A. Ghaffar, Azeema, K. S. Nisar, Fractional calculus of extended Mittag-Leffler function and its applications to statistical distribution, Mathematics, 7 (2019), 14 pages.

[4] M. Arshad, J. S. Choi, S. Mubeen, K. S. Nisar, G. Rahman, A new extension of the Mittag-Leffler function, Commun. Korean Math. Soc., 33 (2018), 549-560. 1, 1

[5] M. Bohner, G. Rahman, S. Mubeen, K. S. Nisar, A further extension of the extended Riemann-Liouville fractional derivative operator, Turkish J. Math., 42 (2018), 2631-2642. 1

[6] P. Cerone, C. T. Lenard, On integral forms of generalized Mathieu series, J. Inequal. Pure Appl. Math., 4 (2003), 1-11. 1

[7] O. Emersleben, Über die Reihe $\sum_{k=1}^{\infty} k /\left(k^{2}+c^{2}\right)^{2}$, Math. Ann., 125 (1952), 165-171. 1

[8] C. Fox, The G and $\mathrm{H}$ functions as symmetrical Fourier kernels, Trans. Amer. Math. Soc., 98 (1961), 395-429. 1, 1

[9] R. Gorenflo, F. Mainardi, Fractional calculus, integral and differential equations of fractional order, in: Fractals and Fractional Calculus in Continuum Mechanics, 1997 (1997), 223-276. 1

[10] R. Hilfer, Application of fractional Calculus in Physics, World Scientific Publishing Co., River Edge, (2000).

[11] C. M. Jiang, F. F. Zhang, T. X. Li, Synchronization and Antisynchronization of N-coupled Fractional-Order Complex Chaotic Systems with Ring Connection, Math. Methods Appl. Sci., 41 (2018), 2625-2638. 1

[12] M. Kamarujjama, O. Khan, Computation of new class of integrals involving generalized Galue type Struve function, J. Comput. Appl. Math., 351 (2019), 228-236. 1

[13] M. Kamarujjama, N. U. Khan, O. Khan, Fractional calculus of generalized p-k-Mittag-Leffler function using MarichevSaigo-Maeda operators, Arab J. Math. Sci., 25 (2019), 156-168. 1

[14] M. Kamarujjama, N. U. Khan, O. Khan, The generalized p-k-Mittag-Leffler function and solution of fractional kinetic equation, J. Anal., 2019 (2019), 18 pages.

[15] M. Kamarujjama, N. U. Khan, O. Khan, J. J. Nieto, Extended type k-Mittag-Leffler function and its applications, Int. J. Appl. Comput. Math., 5 (2019), 14 pages.

[16] O. Khan, N. U. Khan, D. Baleanu, K. S. Nisar, Computable solution of fractional kinetic equations using Mathieu-type series, Adv. Difference Equ., 2019 (2019), 13 pages. 1, 1

[17] A. A. Kilbas, M. Saigo, Fractional calculus of the H-function, Fukuoka Univ. Sci. Rep., 28 (1998), 41-51.

[18] A. A. Kilbas, N. Sebastian, Generalized fractional integration of Bessel function of first kind, Integral Transforms Spec. Funct., 19 (2008), 869-883. 1 
[19] O. L. Marichev, Voletra equation of Mellin convolution type with a horn function in the kernel, Izvestiya Akademii Nauk BSSR Seriya Fiziko-Matematicheskikh Nauk, 1 (1974), 128-129. 1

[20] E. L. Mathieu, Traité de Physique Mathématique, Gauthier-Villars, Paris, (1980). 1

[21] V. N. Mishra, D. L. Suthar, S. D. Purohit, Marichev-Saigo-Maeda fractional calculus operators, Srivastava polynomials and generalized Mittag-Leffler function, Cogent Math., 4 (2107), 11 pages. 4

[22] G. Mittag-Leffler, Sur la Nouvelle Fonction $\mathrm{E}_{\alpha}(x)$, Comptes Rendus de l'Academie des Sciences Paris, 137 (1903), 554-558. 1

[23] K. S. Nisar, D. Baleanu, M. M. Al Qurashi, Fractional calculus and application of generalized Struve function, Springer Plus, 2016 (2016), 13 pages. 1

[24] K. S. Nisar, A. F. Eata, M. Al-Dhaifallah, J. S. Choi, Fractional calculus of generalized k-Mittag-Leffler function and its applications to statistical distribution, Adv. Difference Equ., 2016 (2016), 17 pages. 1

[25] K. S. Nisar, D. L. Suthar, M. Bohra, S. D. Purohit, Generalized fractional integral operators pertaining to the by-product of Srivastava's polynomials and generalized Mathieu Series, Mathematics, 7 (2019), 8 pages. 1

[26] T. R. Prabhakar, A singular integral equation with a generalized Mittag Leffler function in the Kernel, Yokohama Math. J., 19 (1971), 7-15. 1, 1

[27] H. Y. Qin, Z. Y. Gu, Y. L. Fu, T. X. Li, Existence of Mild Solutions and Controllability of Fractional Impulsive Integrodifferential Systems with Nonlocal Conditions, J. Funct. Spaces, 2017 (2017), 11 pages. 1

[28] G. Rahman, S. Mubeen, K. S. Nisar, J. S. Choi, Certain extended special functions and fractional integral and derivative operators via an extended beta function, Nonlinear Functional Analysis and Applications, 24 (2019), 13 pages. 1

[29] G. Rahman, K. S. Nisar, J. S. Choi, S. Mubeen, M. Arshad, Pathway Fractional Integral Formulas Involving Extended Mittag-Leffler Functions in the Kernel, Kyungpook Math. J., 59 (2019), 125-134. 1, 1

[30] M. Saigo, A remark on integral operators involving the gauss hypergeometric functions, Math. Rep. Kyushu Univ., 11 (1977/78), 135-143. 1, 1, 1

[31] M. Saigo, N. Maeda, More generalization of fractional calculus, Transform methods and special functions, in: Transform Methods and Special Functions, 1996 (1996), 386-400. 1, 1, 1

[32] R. K. Saxena, M. Saigo, Certain properties of fractional calculus operators associated with generalized Mittag-Leffler function, Fract. Calc. Appl. Anal., 8 (2005) 141-154. 4

[33] A. K. Shukla, J. C. Prajapati, On a generalization of Mittag-Leffler function and its Properties, J. Math. Anal. Appl., 336 (2007), 797-811. 1

[34] G. Singh, P. Agrwal, S. Araci, M. Acikgoz, Certain fractional calculus formulas involving extended generalized Mathieu series, Adv. Difference Equ., 2018 (2018), 30 pages. 1, 4

[35] H. M. Srivastava, A contour integral involving Fox's H-function, Indian J. Math., 14 (1972), 1-6.

[36] H. M. Srivastava, P. W. Karlsson, Multiple Gaussian hypergeometric series, Ellis Horwood Ltd., Chichester, (1985). 1

[37] H. M. Srivastava, R. K. Saxena, Operators of fractional integration and their applications, Appl. Math. Comput., 118 (2001), 1-52. 1

[38] H. M. Srivastava, Ž. Tomovski, Some problems and solutions involving Mathieu series and its generalizations, JIPAM. J. Inequal. Pure Appl. Math., 5 (2004), 13 pages. 1

[39] Ž. Tomovski, K. Mehrez, Some families of generalized Mathieu-type power series, Math. Inequal. Appl., 20 (2017), 973-986. 1

[40] Ž. Tomovski, T. K. Pogány, Integral expressions for Mathieu-type power series and for the Butzer-Flocke-Hauss function, Fract. Calc. Appl. Anal., 14 (2011), 623-634. 1

[41] Ž. Tomovski, K. Trenčevski, On an open problem of Bai-Ni Guo and Feng Qi, JIPAM. J. Inequal. Pure Appl. Math., 4 (2003), 7 pages. 1

[42] P. G. Wang, C. R. Li, J. Zhang, T. X. Li, Quasilinearization method for first-order impulsive integro-differential equations, Electron. J. Differential Equations, 2019 (2019), 14 pages. 1

[43] A. Wiman, Über den fundamental satz in der theorie der funktionen $\mathrm{E}_{\alpha}(\mathrm{x})$, Acta Mathematica, 29 (1995), 191-201. 1

[44] E. M. Wright, The asymptotic expansion of the generalized hypergeometric function, J. London Math. Soc., 10 (1935), 257-270. 1 